\title{
The role of virulence factors in the outcome of staphylococcal peritonitis in CAPD patients Pasqual Barretti ${ }^{1}$, Augusto C Montelli1 ${ }^{1}$ Jackson EN Batalha1, Jacqueline CT Caramori ${ }^{1}$ and Maria de Lourdes RS Cunha*2
}

\author{
Address: ${ }^{1}$ Department of Internal Medicine, Botucatu School of Medicine, UNESP, Botucatu, Sao Paulo, Brazil and ${ }^{2}$ Department of Microbiology \\ and Immunology, Biosciences Institute, UNESP, Botucatu, Sao Paulo, Brazil \\ Email: Pasqual Barretti - pbarretti@uol.com.br; Augusto C Montelli - acmontelli@uol.com.br; Jackson EN Batalha - jackbatalha@yahoo.com.br; \\ Jacqueline CT Caramori - jteixeir@fmb.unesp.br; Maria de Lourdes RS Cunha* - cunhamlr@ibb.unesp.br \\ * Corresponding author
}

Published: 22 December 2009

BMC Infectious Diseases 2009, 9:212 doi:10.1186/147/-2334-9-2/2
Received: I April 2009

Accepted: 22 December 2009

This article is available from: http://www.biomedcentral.com/I47I-2334/9/2I2

(c) 2009 Barretti et al; licensee BioMed Central Ltd.

This is an Open Access article distributed under the terms of the Creative Commons Attribution License (http://creativecommons.org/licenses/by/2.0), which permits unrestricted use, distribution, and reproduction in any medium, provided the original work is properly cited.

\begin{abstract}
Background: Peritonitis continues to be the most frequent cause of peritoneal dialysis (PD) failure, with an important impact on patient mortality. Gram-positive cocci such as Staphylococcus epidermidis, other coagulase-negative staphylococci (CoNS), and Staphylococcus aureus are the most frequent etiological agents of PD-associated peritonitis worldwide. The objective of the present study was to compare peritonitis caused by $S$. aureus and CoNS and to evaluate the factors influencing outcome.
\end{abstract}

Methods: Records of 86 new episodes of staphylococcal peritonitis that occurred between 1996 and 2000 in the Dialysis unit of a single university hospital were studied (35 due to S. aureus, 24 to $S$. epidermidis and 27 to other CoNS). The production of slime, lipase, lecithinase, nuclease (DNAse), thermonuclease (TNAse), $\alpha$ - and $\beta$-hemolysin, enterotoxins (SEA, SEB, SEC, SED) and toxic shock syndrome toxin-I (TSST-I) was studied in S. aureus and CoNS. Antimicrobial susceptibility was evaluated based on the minimal inhibitory concentration determined by the Etest. Outcome predictors were evaluated by two logistic regression models.

Results: The oxacillin susceptibility rate was $85.7 \%$ for $S$. aureus, $41.6 \%$ for $S$. epidermidis, and $51.8 \%$ for other CoNS $(p=0.001)$. Production of toxins and enzymes, except for enterotoxin $A$ and $\alpha-$ hemolysin, was associated with $S$. aureus episodes $(p<0.001)$, whereas slime production was positive in $23.5 \%$ of CoNS and $8.6 \%$ of $S$. aureus strains ( $p=0.0047)$. The first model did not include enzymes and toxins due to their association with $S$. aureus. The odds of resolution were 9.5 times higher for $S$. epidermidis than for $S$. aureus $(p=0.02)$ episodes, and were similar for $S$. epidermidis and other CoNS $(p=0.8)$. The resolution odds were 68 times higher for non-slime producers ( $P$ $=0.00 \mathrm{I})$ and were not influenced by oxacillin resistance among vancomycin-treated cases $(p=$ $0.89)$. In the second model, the resolution rate was similar for $S$. aureus and $S$. epidermidis $(p=0.70)$, and slime $(p=0.00 \mathrm{I})$ and $\alpha$-hemolysin $(p=0.04)$ production were independent predictors of nonresolution.

Conclusion: Bacterial species and virulence factors rather than antibiotic resistance influence the outcome of staphylococcal peritonitis. 


\section{Background}

Peritonitis continues to be the most frequent cause of peritoneal dialysis (PD) failure [1], and has an important impact on patient mortality [2]. Gram-positive cocci such as Staphylococcus epidermidis, other coagulase-negative staphylococci (CoNS), and Staphylococcus aureus are the most frequent etiological agents of $\mathrm{PD}$-associated peritonitis worldwide [3].

Previous studies comparing the outcome of peritonitis caused by $S$. aureus and CoNS have shown a lower resolution rate and a higher frequency of complications in the former [4-8]. Perez-Fontan et al [2] observed a mortality rate of $15.2 \%$ for $S$. aureus episodes and of only $0.5 \%$ for CoNS episodes. These findings are in contrast to the susceptibility profile observed for CoNS. According to Kim et al [9], the frequency of methicillin-resistance among CoNS increased from $18.4 \%$ in $1992-1993$ to $41.7 \%$ in 2000-2001. Similar results have been reported by other investigators [10]. Previous data from our group showed that oxacillin resistance does not influence the outcome of staphylococcal peritonitis [8].

In addition to species and antibiotic resistance, other factors related to the causal agent may influence the prognosis of peritonitis. Recurrence of CoNS infections is frequently observed and has been suggested to be associated with the presence of a biofilm in the peritoneal catheter [11]. Biofilm formation is related to the production of an extracellular mucoid polysaccharide, called slime, which permits microorganisms to adhere to plastic surfaces [12]. Kristinsson et al [13] reported a higher recurrence rate of peritonitis for slime-positive strains compared to slime-negative ones, whereas Alexander and Rimland [14] did not observe a relationship between slime production and peritonitis outcome. In our unit, slime production was found to be an independent risk factor for the non-resolution of CoNS peritonitis [15].

The production of enzymes and toxins is a well-known fact in Staphylococcus species, particularly S. aureus. Proteases, lipases, nucleases, and collagenases convert tissue components into nutrients, facilitating bacterial growth and invasion [16], while toxic shock syndrome toxin (TSST-1) and enterotoxins have effects such as superantigenicity, pyrogenicity, toxicity and direct damage to endothelial [6].

Although these products are potential virulence factors in staphylococcal PD-associated peritonitis, their influence on the clinical outcome of these infections is unknown. The objective of the present study was to compare the capacity of traditional clinical and bacteriologic and selected virulence factors such as production of slime, enzymes and toxins to predict the outcome of new perito- nitis episodes caused by S. aureus, S. epidermidis and other CoNS.

\section{Methods \\ Data collection and definitions}

The present study was approved by the institutional Ethics Committee. All episodes of continuous ambulatory peritoneal dialysis (CAPD)-associated peritonitis caused by staphylococcal species between January 1996 and December 2000 were reviewed. This period was chosen because a single antibiotic protocol based on the 1996 Update of the International Society for Peritoneal Dialysis [17] was used. After this period, new guidelines were proposed [18] and adopted in our unit. The diagnosis of peritonitis was made when at least two of the following criteria were present: (a) presence of a cloudy peritoneal effluent, (b) abdominal pain, (c) dialysate white cell count higher than $100 / \mu \mathrm{L}$, with at least $50 \%$ polymorphonuclear cells, and (d) positive culture of peritoneal effluent [17].

Only cases considered to be new episodes, i.e., a patient's first peritonitis or an episode diagnosed at least 28 days after completion of the last peritonitis treatment, were included in the study [17]. Thus, 86 of 122 diagnosed staphylococcal peritonitis episodes were analyzed. Exclusion criteria were staphylococcal peritonitis within 28 days prior to presentation, presence of concomitant exit site or tunnel infections, incomplete clinical data, concomitant antibiotic use for other indications, and use of an empirical antibiotic protocol other than the combination of cefazolin and amikacin.

Resolution was defined as the disappearance of signs and symptoms within $96 \mathrm{~h}$ after the beginning of antibiotic therapy and a negative peritoneal fluid culture at least 28 days after treatment completion [7]. Relapse was defined as an episode with the same organism or a negative culture result that occurs within 28 days of completion of antibiotic therapy for a prior episode [17]. Non-resolution was the term used for cases presenting initial non-resolution, relapse, peritoneal catheter removal, or death.

The following information was recorded for each case: (1) episode: date, clinical findings, treatment, outcome (resolution, relapse, catheter removal, or death); (2) presence of diabetes mellitus; (3) demographic data: age, gender, and race (Caucasian, non-Caucasian), dialysis treatment time; (4) exchange system (standard or double bag).

\section{Clinical management}

All episodes were treated according to the local protocol adapted from the third report of the Ad Hoc Advisory Committee on Peritonitis Management for staphylococcal episodes [17]. Patients were treated within $24 \mathrm{~h}$ of the onset of the first clinical signs or symptoms and antibiotic 
therapy was started with 500 or $750 \mathrm{mg} / \mathrm{L}$ cefazolin, ip (patients with urine volume $>500 \mathrm{~mL} / 24 \mathrm{~h}$ ) and $250 \mathrm{mg} /$ $\mathrm{L}$ amikacin, ip, as loading dose, followed by 500 or 750 $\mathrm{mg} / \mathrm{L}$ cefazolin and $2 \mathrm{mg} / \mathrm{kg}$ amikacin per day in the last PD bag. Therapy was evaluated as soon as the culture results were available. For oxacillin-susceptible cocci, cefazolin was maintained, whereas for oxacillin-resistant cocci cefazolin was replaced with $1 \mathrm{~g} / \mathrm{L}$ vancomycin, ip, administered at intervals of 5 (patients with urine volume $>500 \mathrm{~mL} / 24 \mathrm{~h}$ ) or 7 days. Amikacin was discontinued in both cases. Vancomycin was also administered to patients who presented no clinical improvement within the first $96 \mathrm{~h}$ of antibiotic treatment, although the microbiological tests revealed oxacillin susceptibility. The duration of antibiotic therapy was 14 days for CoNS episodes and 21 days for $S$. aureus episodes.

\section{Microbiological tests}

Microbiological samples were stored in a culture collection. The isolates obtained from clinical specimens were seeded onto blood agar and gram-stained to confirm purity and to determine morphology and specific color. The isolates were then submitted to catalase and coagulase tests. CoNS were identified using the simplified biochemical test scheme proposed by Kloos and Schleifer [19] and Kloos and Bannerman [20].

In vitro susceptibility was evaluated based on the minimal inhibitory concentration determined by the E-test (AB Biodisk, Solna, Sweden), a quantitative method that uses a transparent strip of inert plastic containing drug concentrations ranging from 0.002 to $256 \mu \mathrm{g} / \mathrm{mL}$. The proportion of strains susceptible to each drug was defined based on the 2005 CLSI breakpoints [21]. Strains presenting intermediate values were considered to be resistant.

\section{Determination of the production of pathogenic factors Slime}

Slime production was evaluated according to Christensen et al [22]. Colonies of CoNS isolated on blood agar were inoculated into tubes measuring $12.0 \times 75.0 \mathrm{~mm}$ and containing $2.0 \mathrm{~mL}$ trypticase soy broth (TSB) and incubated for $48 \mathrm{~h}$ at $37^{\circ} \mathrm{C}$. Next, $1.0 \mathrm{~mL} 0.4 \%$ trypan blue or toluidine blue $\mathrm{O}$ solution was added to the tubes. After gentle shaking to guarantee staining of the material adhered to the inner surface of the tubes, the dye was discarded. A positive result was defined as the presence of a layer of stained material adhered to the inner wall of the tubes. The presence of a colored ring only at the liquid-air surface was classified as negative.

\section{Alpha- and beta-hemolysin}

Production of $\alpha$ - and $\beta$-hemolysin or cytolytic toxins was determined on plates containing blood agar base supplemented with 5\% rabbit blood and 5\% sheep blood, respectively. The plates were incubated for $24 \mathrm{~h}$ at $37^{\circ} \mathrm{C}$. The formation of hemolysis zones around the isolated colonies indicated a positive result.

\section{Lipase and lecithinase}

Lipolytic activity was determined on plates containing blood agar base enriched with $0.01 \% \mathrm{CaCl}_{2}: 2 \mathrm{H}_{2} \mathrm{O}$ and $1 \%$ Tween 80 . A positive result was defined as the formation of opacity around the colony after incubation for 18 $\mathrm{h}$ at $37^{\circ} \mathrm{C}$, followed by incubation at room temperature for $24 \mathrm{~h} \mathrm{[23]}$. The production of lecithinase was evaluated using Baird-Parker medium. The formation of an opaque halo around the colony indicated a positive result [24].

\section{DNAse and TNAse}

Nuclease (DNAse) and thermonuclease (TNAse) were determined by the metachromatic toluidine blue $\mathrm{O}$ agar diffusion-DNA technique according to Lachica et al [25]. For the detection of DNAse, supernatants were obtained from CoNS cultures in BHI broth previously incubated for $24 \mathrm{~h}$ at $37^{\circ} \mathrm{C}$ and centrifuged at $8000 \mathrm{~g}$ for $10 \mathrm{~min}$ at $4^{\circ} \mathrm{C}$. The culture supernatant was first heated in a water bath for $20 \mathrm{~min}$ and then placed in the wells for the detection of TNAse. Nuclease (DNAse and TNAse) activity was determined by measuring the diameter of pink halos ( $\mathrm{mm}$ ) formed on the medium. Positive results were interpreted by comparison of the halos with those obtained for a standard DNAse- and TNAse-positive $S$. aureus strain (ATCC 25923). Culture supernatants obtained by the sac culture method of Donnelly et al [26], as described below, were also tested for DNAse and TNAse production.

\section{Enterotoxins and toxic shock syndrome toxin-I}

The sac culture method for toxin production [26] was used to determine the toxigenic profile of the strains. Dialysis sacs filled with $50 \mathrm{~mL}$ double-concentrated BHI broth were placed in U-shaped Erlenmeyer flasks and autoclaved for $15 \mathrm{~min}$ at $121^{\circ} \mathrm{C}$. A loopful of organisms was added to $18 \mathrm{~mL}$ sterile $0.2 \mathrm{M}$ phosphate buffer in $0.9 \%$ $\mathrm{NaCl}, \mathrm{pH}$ 7.4. After incubation for $24 \mathrm{~h}$ at $37^{\circ} \mathrm{C}$ on a shaker at $200 \mathrm{rpm}$, the cultures were centrifuged at $8000 \mathrm{~g}$ for $10 \mathrm{~min}$ at $4^{\circ} \mathrm{C}$ and the supernatants obtained were stored at $-20^{\circ} \mathrm{C}$ until the time of use. The extracellular products were detected by reverse passive latex agglutination (RPLA) according to Shingaki et al [27], using the SET-RPLA-T900 and TST-RPLA-TD940 kits (Oxoid Diagnostic Reagents) for the detection of enterotoxins A (SEA), B (SEB), C (SEC) and D (SED) and TSST-1, respectively. Culture supernatants were first treated with $5 \%(\mathrm{v} / \mathrm{v})$ normal rabbit serum or $5 \%$ purified rabbit IgG to block nonspecific reactions. Samples that presented nonspecific reactions even after this treatment were filtered through a Millipore membrane $(8.0 \mu \mathrm{m})$ and, if necessary, diluted $1: 10$ with $0.02 \mathrm{M}$ phosphate buffer in $0.9 \% \mathrm{NaCl}, \mathrm{pH} 7.4$. A positive reaction was classified as $(+),(++)$ and $(+++)$ 
according to the agglutination pattern described by the manufacturer of the kit. The formation of a rose button was interpreted as a negative result.

\section{Statistical analysis}

Continuous variables were compared using the unpaired t-test or nonparametric Mann-Whitney U-test. Binary variables were compared by the chi-square or Fisher's exact test. Multivariate analysis by logistic regression was used to test for factors that independently predicted the outcome of a peritonitis episode, with outcome being classified into two mutually exhausted and exclusive results (resolution or non-resolution). A model was adopted that would incorporate the effect of all factors-interest and control - on the outcome of infection. All baseline demographic, clinical, and microbiological variables, including age, gender, diabetic status, dialysis duration, exchange system, use of vancomycin, Staphylococcus species, oxacillin susceptibility and pathogenic factor production, were included in the model. A p value less than 0.05 was considered to be significant.

\section{Results}

Eighty-six new CAPD-associated staphylococcal peritonitis episodes occurred in 63 patients between 1996 and 2000. Forty-three of the patients were females, 39 were Caucasians, and 28 had diabetes. The distribution of patients according to age was as follows: birth to 20 years $(\mathrm{n}=4), 21$ to 40 years $(\mathrm{n}=12), 41$ to 59 years $(\mathrm{n}=22)$, and 60 years or older $(n=22)$. Treatment time on CAPD was less than one year in 28 patients and longer than one year in 35. Forty-four patients used a double bag system and 19 used a standard bag exchange system.

\section{Microbiological investigation}

Thirty-five episodes were caused by $S$. aureus and 51 by CoNS. Among CoNS, S. epidermidis was the most frequent species ( 24 cases), followed by $S$. haemolyticus (11 cases) and other species (16 cases) (Table 1 ).

Table I: Causative agent of the 86 new episodes of peritonitis caused by $S$. aureus and coagulase-negative staphylococci.

\begin{tabular}{lc}
\hline Microorganism & No. of strains (\%) \\
\hline S. aureus & 35 \\
CoNS & $51(100)$ \\
S. epidermidis & $24(47)$ \\
S. haemolyticus & $11(21.5)$ \\
S. warneri & $5(9.8)$ \\
S. hominis & $5(9.8)$ \\
S. xylosus & $2(3.9)$ \\
S. cohnii & $2(3.9)$ \\
S. simulans & $1(1.9)$ \\
S. lugdunensis & $1(1.9)$ \\
\hline
\end{tabular}

CoNS: coagulase-negative staphylococci.
Oxacillin susceptibility was observed in 30 (85.7\%) of the 35 episodes due to $S$. aureus, in $10(41.7 \%)$ of the 24 episodes due to $S$. epidermidis, and in 12 (44.4\%) of the 27 episodes due to other CoNS $(p=0.0002)$. Five cases of intermediate susceptibility were detected among CoNS and one among $S$. aureus strains.

Positive slime production was observed in three cases due to $S$. aureus $(8.6 \%)$ and in nine $(17.6 \%)$ due to CoNS ( $p=$ $0.345)$, including $S$. epidermidis in four, $S$. haemolyticus in two, S. warneri in two, and S. lugdunensis in one.

With respect to toxigenic profile, 25 (71.4\%) of the 35 S. aureus strains were toxin producers, whereas only seven (13.7\%) of the 51 CoNS strains produced some type of toxin $(p<0.00001)$. The rate of enzyme production was higher in $S$. aureus strains than in S. epidermidis or other CoNS, except for $\alpha$-hemolysin whose production was similar in all strains. The rates of toxin and enzyme production by $S$. aureus, S. epidermidis and other CoNS species are shown in Table 2.

\section{Clinical outcome}

Overall, 57 (66.3\%) episodes were resolved, 14 (16.3\%) relapsed, $12(13.9 \%)$ required removal of the catheter, and three (3.5\%) progressed to death. Among the $35 \mathrm{~S}$. aureus cases, 17 (48.6\%) were resolved, eight (22.8\%) relapsed, seven $(20 \%)$ required catheter removal, and three $(8.6 \%)$ progressed to death. Regarding CoNS episodes, $40(78.4 \%)$ were resolved, six (11.8\%) relapsed, and five $(9.8 \%)$ required catheter removal. Among these 11 non-resolved episodes, seven were due to $S$. epidermidis, two to $S$. haemolyticus, and two to other CoNS. There were significantly more CoNS cases resolved than $S$. aureus episodes $(\mathrm{p}<0.001)$.

There were 52 episodes involving oxacillin-susceptible strains, $32(61.5 \%)$ of them were resolved, $10(19.2 \%)$ relapsed, and $10(19.2 \%)$ required catheter removal. Thirty-four infections were caused by oxacillin-resistant strains, 20 (58.8\%) of them were resolved, nine $(26.5 \%)$ relapsed, and five (14.7\%) required catheter removal. The resolution rate was similar for oxacillin-susceptible and resistant strains $(\mathrm{p}=0.9713)$.

Vancomycin was used in 56 episodes, 18 caused by $S$. aureus and 38 by CoNS. This antibiotic was prescribed because of bacterial resistance in 28 cases, lack of improvement in 20, and other undefined causes in eight. The time between diagnosis and the first vancomycin dose was $3.7 \pm 1.7$ days for $S$. aureus episodes and $3.9 \pm 1.2$ days for CoNS episodes ( $\mathrm{p}=0.793)$.

Two regression models were constructed. In Model 1 slime production was the only pathogenic factor, whereas 
Table 2: Rates of toxin and enzyme production by S. aureus, S. epidermidis and other coagulase-negative staphylococci strains isolated from 86 new episodes of peritonitis.

\begin{tabular}{|c|c|c|c|c|}
\hline & S. aureus & S. epidermidis & Other CoNS & $\mathbf{p}$ \\
\hline \multicolumn{5}{|l|}{ Toxins } \\
\hline SEA & 4 (II.4) & I (4.I) & $-(-)$ & 0.15 \\
\hline SEB & $12(34.3)$ & $-(-)$ & $2(7.4)$ & 0.0006 \\
\hline SEC & $8(22.8)$ & $-(-)$ & $6(22.2)$ & $0.04^{*}$ \\
\hline SED & $-(-)$ & $-(-)$ & $-(-)$ & - \\
\hline TSST-I & $12(34.3)$ & $I(4.1)$ & $-(-)$ & 0.0008 \\
\hline \multicolumn{5}{|l|}{ Enzymes } \\
\hline$\alpha$-Hemolysin & $17(48.6)$ & $8(33.3)$ & $8(29.8)$ & 0.26 \\
\hline$\beta$-Hemolysin & $29(82.3)$ & $6(25)$ & $7(25.9)$ & $<0.00001$ \\
\hline Lipase & 34 (97.I) & $4(16.7)$ & $5(18.5)$ & $<0.00001$ \\
\hline Lecithinase & $34(97.1)$ & $2(8.3)$ & $6(22.2)$ & $<0.00001$ \\
\hline DNAse & 34 (97.I) & $-(-)$ & $4(14.8)$ & $<0.00001$ \\
\hline TNAse & $34(97.1)$ & $-(-)$ & $4(14.8)$ & $<0.00001$ \\
\hline
\end{tabular}

CoNS: coagulase-negative staphylococci; SEA, SEB, SEC, and SED: enterotoxins A, B, C and D, respectively; TSST-I: toxic shock syndrome toxin; * S. aureus vs. S. epidermidis and other CoNS vs. S. epidermidis.

in Model 2 the production of toxins and enzymes was included. Only lecithinase, $\alpha$-hemolysin and TNAse were included since associations were observed between lecithinase and lipase, $\alpha$-hemolysin and $\beta$-hemolysin, and TNAse and DNAse $(p=0.001)$. All toxins were included, except for toxin $\mathrm{D}$ which was not produced by any of the strains. Since an interaction effect was observed between S. aureus species and oxacillin susceptibility $(\mathrm{p}=0.01)$, the influence of oxacillin susceptibility was evaluated at each vancomycin treatment co-variable level in both models.

In Model 1 (Table 3), controlling for co-variables, the odds of resolution was not influenced by host factors such as age, gender, diabetes, exchange system, or CAPD treatment time. The odds of resolution were 9.5 times higher for $S$. epidermidis than for $S$. aureus episodes $(p=0.0263)$, whereas similar resolution odds were observed for S. epidermidis and the other CoNS $(p=0.085)$. Among strains isolated from infections treated with vancomycin, no significant difference was observed between oxacillin-susceptible and -resistant strains $(\mathrm{p}=0.89)$. In contrast, among strains isolated from infections not treated with vancomycin, there was a significant difference between strains susceptible and resistant to oxacillin ( $p=0.0113)$. In this case, the chance of cure of infections caused by oxacillin-susceptible strains was 137 times higher than that of

Table 3: Odds comparison of peritonitis resolution by logistic regression analysis (Model I).

\begin{tabular}{|c|c|c|c|}
\hline Factor & Log (Odds) & $\mathbf{p}$ & $\begin{array}{l}\text { Odds ratio } \\
\text { (95\% Cl) }\end{array}$ \\
\hline Age (birth to 20 years/>60 years) & -0.0510 & 0.96 & \\
\hline Age ( 21 to 40 years $/>60$ years) & 1.7097 & 0.09 & \\
\hline Age ( 4 I to 59 years $/>60$ years) & 0.7511 & 0.84 & \\
\hline Gender (male/female) & 1.2523 & 0.19 & \\
\hline Race (Caucasian/non-Caucasian) & -0.0818 & 0.92 & \\
\hline Diabetes mellitus (no/yes) & -0.6709 & 0.12 & \\
\hline System (standard/double bag) & 1.2585 & 0.22 & \\
\hline \multicolumn{4}{|l|}{ Treatment time on CAPD } \\
\hline$<$ I year & 0.2074 & 0.89 & \\
\hline$>$ I year & 0.9547 & 0.21 & \\
\hline \multicolumn{4}{|l|}{ Treatment with vancomycin } \\
\hline Oxacillin susceptible/oxacillin resistant & -0.1070 & 0.89 & \\
\hline \multicolumn{4}{|l|}{ No treatment with vancomycin } \\
\hline Oxacillin susceptible/oxacillin resistant & 1.9440 & 0.01 & $137(3.0 ; 6,202.4)$ \\
\hline \multicolumn{4}{|l|}{ Etiological agent } \\
\hline S. epidermidis/S. aureus & 2.2548 & 0.02 & $9.5(1.3 ; 69.6)$ \\
\hline Other CoNS/S. epidermidis & -1.6113 & 0.08 & \\
\hline Slime production (no/yes) & 4.2139 & 0.001 & $68(5.0 ; 914.8)$ \\
\hline
\end{tabular}

CoNS: coagulase-negative staphylococci; $\mathrm{Cl}$ : 95\% Confidence Interval for the true Odds Ratio. 
infections caused by resistant strains. With respect to slime production, the chance of cure of infections caused by non-producers was estimated to be up to 68 times higher than that of infections caused by slime producers $(\mathrm{p}=0.0015)$.

As observed for the first model, in Model 2 (Table 4) resolution odds were not influenced by host factors. Among strains isolated from infections treated with vancomycin, no significant difference was observed between those susceptible and resistant to oxacillin ( $\mathrm{p}=0.1523$ ), whereas there was a significant difference among strains not treated with vancomycin $(\mathrm{p}=0.0039)$. With respect to slime production, the chance of cure of infections caused by non-producers was estimated to be 184 times higher than that of infections caused by producers $(\mathrm{p}=0.0012)$. In contrast to Model 1, S. aureus did not differ from S. epidermidis in terms of the probability of peritonitis resolution ( $\mathrm{p}=0.7014)$, whereas the chance of cure of infections caused by other CoNS species was estimated to be 46 times higher than that of infections caused by $S$. epidermidis ( $\mathrm{p}=0.0175)$. Alpha-hemolysin production was an independent predictor of resolution odds, with episodes caused by non-producers presenting an 8.2 times higher chance of resolution than those caused by producers $(\mathrm{p}=$
0.0423). No significant effects on the probability of peritonitis resolution were observed for the remaining enzymes and toxins.

\section{Discussion}

In the present study we investigated new episodes of staphylococcal peritonitis in PD patients and compared episodes caused by S. aureus, S. epidermidis and other CoNS, focusing on the role of virulence factors in peritonitis outcome. CoNS were the most frequent etiological agent, in agreement with other studies. In addition to S. epidermidis, seven other CoNS species were identified, the most predominant being $S$. haemolyticus. This is an important finding since studies regarding other CoNS are scarce in the literature.

The resolution rate of episodes caused by $S$. aureus was lower than that of infections caused by CoNS. Similar findings have been reported in the prospective studies of Bunke et al [5] and Peacock et al [6], and in the recent retrospective study of Davenport [1]. Since the rate of oxacillin resistance was higher among CoNS strains than among $S$. aureus strains, a contribution of drug resistance is unlikely. In fact, our results showed no difference in reso-

Table 4: Odds comparison of peritonitis resolution by logistic regression analysis (Model 2).

\begin{tabular}{|c|c|c|c|}
\hline Factor & Log (Odds) & $\mathbf{p}$ & $\begin{array}{l}\text { Odds ratio } \\
\text { (95\% Cl) }\end{array}$ \\
\hline Age (birth to 20 years/>60 years) & 0.0602 & 0.97 & \\
\hline Age ( 2 I to 40 years $/>60$ years $)$ & 4.2565 & 0.06 & \\
\hline Age ( 4 I to 59 years $/>60$ years) & 1.9474 & 0.09 & \\
\hline Gender (male/female) & 1.2461 & 0.37 & \\
\hline Race (Caucasian/non-Caucasian) & -1.3322 & 0.21 & \\
\hline Diabetes mellitus (no/yes) & -0.6711 & 0.47 & \\
\hline System (standard/double bags) & 2.3397 & 0.12 & \\
\hline \multicolumn{4}{|l|}{ Treatment time on CAPD } \\
\hline$<1$ year & 0.0531 & 0.98 & \\
\hline$>1$ year & -0.3045 & 0.77 & \\
\hline \multicolumn{4}{|l|}{ Treatment with vancomycin } \\
\hline Oxacillin susceptible/oxacillin resistant & 1.6316 & 0.15 & \\
\hline \multicolumn{4}{|l|}{ No treatment with vancomycin } \\
\hline Oxacillin susceptible/oxacillin resistant & 10.0189 & 0.004 & $23,906(25.4 ; \exp \{16.9274\})$ \\
\hline \multicolumn{4}{|l|}{ Etiological agent } \\
\hline S. epidermidis S. aureus & $-|.648|$ & 0.70 & \\
\hline Other CoNS/S. epidermidis & 3.8238 & 0.017 & $46(2.0 ; 1,069.4)$ \\
\hline Slime production (no/yes) & 5.2149 & 0.001 & $184(7.8 ; 4,354.2)$ \\
\hline \multicolumn{4}{|l|}{ Enzyme production (no/yes) } \\
\hline$\alpha-$ Hemolysin & 2.1092 & 0.04 & $8.2(1.1 ; 63.1)$ \\
\hline TNAse & 2.0500 & 0.28 & \\
\hline Lecithinase & 3.5545 & 0.06 & \\
\hline \multicolumn{4}{|l|}{ Toxin production (no/yes) } \\
\hline Enterotoxin A & 2.3914 & 0.26 & \\
\hline Enterotoxin B & 0.5669 & 0.13 & \\
\hline Enterotoxin $\mathrm{C}$ & 4.0501 & 0.06 & \\
\hline TSST-I & 1.3694 & 0.48 & \\
\hline
\end{tabular}


lution rates between episodes caused by oxacillin-susceptible and oxacillin-resistant strains.

All patients received an intermittent regimen of cefazolin plus amikacin as initial treatment. Some investigators $[28,29]$ argue that the continuous addition of betalactams to the PD fluid is more effective for the treatment of CoNS infections by overcoming moderate bacterial resistance. Since a higher resolution rate was observed for CoNS episodes compared to $S$. aureus episodes and the number of strains presenting moderate resistance to oxacillin was low, it is unlikely that the antibiotic regimen has influenced the results.

Regression analysis using the two models showed no influence of age, race, gender, CAPD treatment time, diabetes, or exchange system on the progression of peritonitis. Similar results have been reported by Krishnan et al [7], except for the influence of race and dialysis treatment time.

Slime production was independently associated with non-resolution of peritonitis. This finding agrees with data on CoNS infections previously published by our group [15] and with the results of Kristinsson et al [13]. The latter authors proposed that slime production may promote bacterial adherence to catheters, facilitating colonization and peritonitis relapse. Thus, slime production might be a virulence factor and simultaneously worsens the response to infection, protecting bacterial cells from the host's natural defense mechanisms and from the action of antibiotics.

In contrast to Model 1, no difference in the probability of peritonitis resolution was observed between $S$. aureus and S. epidermidis when Model 2 was used. This finding might be explained by the fact that inclusion of enzymes and toxins in the model permitted the control for the effect of species factor on these bacterial products. In other words, the species effect observed in the first model for $S$. aureus episodes was not separated from the pathogenicity factors included in the second model. In addition, whereas the chance of cure of $S$. epidermidis episodes compared to other CoNS infections tended to be lower in the first model, the second model showed a significantly lower probability of cure of $S$. epidermidis episodes, an independent outcome not influenced by toxin or enzyme production.

Among the virulence factors studied, only $\alpha$-hemolysin production was significantly and independently associated with a higher probability of non-resolution. Recent data published by Haslinger-Löffler et al [30] suggest that $\alpha$-hemolysin plays a specific role in the pathogenesis of peritonitis. Using cultured human peritoneal mesothelial cells, these authors showed that the $S$. aureus subgroup characterized as invasive and $\alpha$-hemolysin producing induced caspase-independent cell death. Unlike S. aureus, no cytotoxic effects were triggered by any of the S. epidermidis strains which were noninvasive and did not produce $\alpha$-hemolysin. These findings, together with our results, suggest that this enzyme plays a pathogenic role in PDassociated peritonitis. Since mesothelial cells participate in the early host defense against infections [31], damage caused by $\alpha$-hemolysin may contribute to the poor course of $S$. aureus peritonitis.

This study has some limitations, particularly the small number of cases, which reduce its statistical power and prevented the separate analysis of $S$. aureus and CoNS peritonitis episodes. Further studies with bigger number of cases are necessary to overcome this limitation and to confirm the present results.

\section{Conclusions}

In conclusion, host factors, as well as dialysis treatment time and exchange system, probably have little or no influence on the response to PD-associated peritonitis treatment. However, the prognosis of these infections is strongly influenced by characteristics of the causative agent such as species and virulence factors. Although these factors may act in concert, our data suggest that slime and $\alpha$-hemolysin production independently contribute to poor peritonitis outcome.

\section{Competing interests}

The authors declare that they have no competing interests.

\section{Authors' contributions}

$\mathrm{PB}$ participated in the design and coordination of the study and in the collection of the clinical data, analyzed the data, and wrote and revised the manuscript. ACM participated in the design of the study and performed the Etest. JENB performed the microbiological tests. JCTC participated in the collection of the clinical data and contributed to the design of the study. MLRSC participated in the coordination of the study, supervised the laboratory work, reviewed and approved the analyses, and contributed to the writing and revision of the paper. All authors read and approved the final manuscript.

\section{Acknowledgements}

This study was supported by Fundação de Amparo à Pesquisa do Estado de São Paulo (FAPESP). We are grateful to Janete Soares for technical assistance and to Dr Luis Cuadrado Martin for his suggestions.

\section{References}

I. Davenport A: Peritonitis remains the major clinical complication of peritoneal dialysis: The London, UK, Peritonitis Audit 2002-2003. Perit Dial Int 2009, 29:297-302.

2. Perez Fontan M, Rodriguez-Carmona A, Garcia-Naveiro R, Rosales $M$, Villaverde P, Valdes F: Peritonitis-related mortality in 
patients undergoing chronic peritoneal dialysis. Perit Dial Int 2005, 25:274-84.

3. Troidle LK, Finkelstein FO: Treatment and outcome of CPDassociated peritonitis. Ann Clin Microbiol Antimicrob 2006, 5:6-25.

4. Troidle L, Gorban-Brennan N, Kliger A, Finkeltein F: Differing outcomes of gram-positive and gram-negative. Am J Kidney Dis 1998, 32:623-628.

5. Bunke CM, Brier ME, Golper TA: Outcomes of single organism peritonitis in peritoneal dialysis: gram negatives versus gram positives in the Network 9 Peritonitis Study. Kidney Int 1997, 52:524-529.

6. Peacock SJ, Howe PA, Day NP, Crook DW, Winearls CG, Berendt AR: Outcome following staphylococcal peritonitis. Perit Dial Int 2000, 20:215-219.

7. Krishnan M, Thodis E, Ikonomopoulos D, Vidgen E, Chu M, Bargman JM, Vas SI, Oreopoulos DG: Predictors of outcome following bacterial peritonitis in peritoneal dialysis. Perit Dial Int 2002, 22:573-58I.

8. de Lourdes Ribeiro de Souza da Cunha M, Montelli AC, Fioravante AM, Neves Batalha JE, Texeira Caramori JC, Barretti P: Predictive factors of outcome following staphylococcal peritonitis in continuous ambulatory peritoneal dialysis. Clin Nephrol 2005, 64:378-382.

9. Kim DK, Yoo TH, Ryu DR, Xu ZG, Kim HJ, Choi KH, Lee HY, Han DS, Kang SW: Changes in causative organisms and their antimicrobial susceptibilities in CAPD peritonitis: a single center's experience over one decade. Perit Dial Int 2004, 24:424-432.

10. Holley JL, Bernadini J, Johnston JR, Piraino B: Methicillin-resistant staphylococcal Infections in an outpatient peritoneal dialysis program. Am J Kidney Dis 1990, 16: I $42-146$.

11. Kristinsson KG, Spencer RC, Hastings JGM, Brown CB: Slime production by coagulase negative Staphylococci - a major virulence factor? Contr Nephrol 1987, 57:79-84.

12. Vogel L, Sloos JH, Spaargaren J, Suiker I, Dijkshoorn L: Biofilm production by Staphylococcus epidermidis isolates associated with catheter related bacteremia. Diagn Microbiol Infect Dis 2000, 36:|39-4|.

13. Kristinsson KG, Spencer RC, Brown CB: Clinical importance of production of slime by coagulase negative staphylococci in chronic ambulatory peritoneal dialysis. J Clin Pathol 1986, 39: $117-118$.

14. Alexander W, Rimland D: Lack of correlation of slime production with pathogenicity in continuous ambulatory peritoneal dialysis peritonitis caused by coagulase negative staphylococci. Diagn Microbiol Infect Dis 1987, 8:215-220.

15. de Lourdes Ribeiro de Souza da Cunha M, Caramori JC, Fioravante AM, Batalha JE, Montelli AC, Barretti P: Significance of slime as virulence factor in coagulase-negative staphylococcus peritonitis in CAPD. Perit Dial Int 2004, 24:191-193.

16. Dingues MM, Orwin PM, Schlievert M: Exotoxins of Staphylococcus aureus. Clin Microb Reviews 2000, 13:16-34.

17. Keane WF, Alexander SR, Bailie GR, Boeschoten E, Gokal R, Golper TA, Holmes CJ, Huang CC, Kawaguchi Y, Piraino B, Riella M, Schaefer $\mathrm{F}$, Vas S: Peritoneal dialysis-related peritonitis treatment recommendations: 1996 update. Perit Dial Int 1996, 16:557-573.

18. Keane WF, Bailie GR, Boeschoten E, Gokal R, Golper TA, Clifford JH, Kawaguchi $Y$, Piraino B, Riella M, Vas S: Adult peritoneal dialysisrelated peritonitis treatment recommendations: 2000 update. Perit Dial Int 2000, 20:396-4II.

19. Kloos WE, Schleifer KH: Simplified scheme for routine identification of human Staphylococcus species. J Clin Microbiol 1975, I:82-88.

20. Kloos WE, Bannerman TL: Staphylococcus and Micrococcus. In Manual of Clinical Microbiology Edited by: Murray PR, Baron EJ, Pfaller MA, Tenover FC, Yolken RH. Washington, American Society Microbiology; 1995:282-298.

21. Clinical and Laboratory Standards Institute: Performance standards for antimicrobial susceptibility testing: CLSI approved standard MI00-SI5. Wayne PA USA 2005.

22. Christensen GD: Adherence of slime-producing strains of Staphylococcus epidermidis to smooth surfaces. Infect Immun I982, 37:318-326.

23. Jessen O, Faber V, Rosendal K, Eriksen KR: Some properties of Staphylococcus aureus, possibly related to pathogenicity. Part
I. A study of 446 strains from different types of human infection. Acta Pathol Microbiol Scand 1959, 47:316-326.

24. Matos JE, Harmon RJ, Langlois BE: Lecithinase reaction of Staphylococcus aureus strains of different origin on Baird-Parker medium. Lett Appl Microbiol 1995, 21:334-335.

25. Lachica RUF, Genigeorgis C, Hoeprich PD: Metachromatic agardiffusion methods for detecting staphylococcal nuclease activity. Appl Microbiol 197I, 21:585-587.

26. Donnelly CB: Serological identification of enterotoxigenic staphylococci from cheese. Appl Microbiol 1967, 15:1382-1387.

27. Shingaki MH, Igarashi H, Fujikawa $H$, Ushioda H, Terayama T, Sakai S: Study on reversed passive latex agglutination for the detection of staphylococcal enterotoxins A-C. Annu Rep Tokyo Metr Res Lab Publ Hith 1981, 32:128-131.

28. Gruer LD, Bartlett R, Ayliffe GA: Species identification and antibiotic sensitivity of coagulase-negative staphylococci from CAPD peritonitis. J Antimicrob Chemother 1984, I 3:577-583.

29. Golper T: Intermittent versus continuous antibiotics for PDrelated peritonitis. Perit Dial Int 1997, 17:II-12.

30. Haslinger-Loffler B, Wagner B, Bruck M, Strangfeld K, Grundmeier M, Fischer U, Volker W, Peters G, Schulze-Osthoff K, Sinha B: Staphylococcus aureus induces caspase-independent cell death in human peritoneal mesothelial cells. Kidney Int 2006, 70:1089-1098.

31. Glik A, Douvdevani A: Tlymphocytes: the "cellular" arm of acquired immunity in the peritoneum. Perit Dial Int 2006, 26:438-448

\section{Pre-publication history}

The pre-publication history for this paper can be accessed here:

\section{http://www.biomedcentral.com/1471-2334/9/212/pre} pub
Publish with Bio Med Central and every scientist can read your work free of charge

"BioMed Central will be the most significant development for disseminating the results of biomedical research in our lifetime. "

Sir Paul Nurse, Cancer Research UK

Your research papers will be:

- available free of charge to the entire biomedical community

- peer reviewed and published immediately upon acceptance

- cited in PubMed and archived on PubMed Central

- yours - you keep the copyright 